\title{
Mobility Deficit - Rehabilitate, An Opportunity for Functionality
}

\author{
Gorete Reis ${ }^{1,2(\triangle)}$ (D), Patrícia Páscoa Pereira ${ }^{3}$ (D), Lena Sabino ${ }^{4}$, \\ and Maria José Bule ${ }^{1}$ (D) \\ ${ }^{1}$ University of Évora, Évora, Portugal \\ greis@uevora.pt \\ 2 Research Group AgeingC, Cintesis - Center for Health Technology and \\ Services Research, FMUP, Investigator POCTEP 0445_4IE_4_P, Porto, Portugal \\ ${ }^{3}$ Polyvalent Intensive Care Unit of Hospital Espírito Santo, Évora, Portugal \\ ${ }^{4}$ Local Health Unit of Baixo Alentejo, Beja, Portugal
}

\begin{abstract}
There are many pathological conditions that cause mobility deficits and that ultimately influence someone's autonomy. To overcome these difficulties implies the patient's involvement and professional action, namely from a rehabilitation nurse, whose target is to capacitate the patient. The person can be capacitated and reach quality of life through organized and systematic rehabilitation programs, which can result in health gains. Aims: to evaluate patients with mobility deficits functional status; to implement a Rehabilitation Nursing intervention plan; to monitor health gains through mobility deficits rehabilitation; Method: Cross-sectional study, action research approximation. Nonprobability sampling, 9 patients admitted at an Intensive Care Unit and at a Stroke Unit who fulfilled the inclusion criteria. The intervention plan included two moments of formal evaluation: the initial (T1) and the final (T2) that relied on specific instruments. Results: improvement and solving of rehabilitation nursing diagnoses, increased balance and better transferring ability. Data suggest that the implementation of an intervention plan decreased the level of dependence. Conclusion: Early intervention and the implementation of a nursing rehabilitation intervention plan results in health gains (direct or indirect), decreases the risk of developing Pressure Ulcers (PU) and the risk of developing a situation of immobility that affects patients' autonomy and quality of life.
\end{abstract}

Keywords: Rehabilitation Nursing $\cdot$ Mobility limitation $\cdot$ Daily activities

\section{Introduction}

The admission at highly differentiated hospital units often forces the patients to a therapeutic rest that naturally evolves to mobility restriction and consequently to its negative effects. To understand the short- and long-term effects of immobility of admitted patients in a post critical situation requires the development of specialized care, focused on self-care. Given this scenario, an early nursing rehabilitation approach is crucial for optimizing patients' functional status and for reducing their inabilities. The problem here studied focuses on dependence, specifically concerning affected 
mobility that restrains the performance of daily activities (DA). The main effects of immobility are: decreased functional mobility, decreased lean muscle mass and difficulty in recovering the functional status prior to hospital admission [1]. The loss of mobility affects the capacity of the individual to remain physically independent, to perform daily activities, resulting in a reduction of his/her quality of life [2].

Current literature highlights that the rehabilitation process after a stroke should start as early as possible [3, 4] as it is considered a major condition for recovery [5]. 30\% of stroke survivors are estimated to develop some level of dependence that prevents them to perform basic daily activities [6]. Treatment at Intensive Care Units (ICU) and the period after a critical medical episode are also associated with increased physical and psychological morbidity [7]. When we focus on the patient functional rehabilitation, after patients, hemodynamic and respiratory stabilization, early rehabilitation has been seen as a positive intervention that works as an important treatment and modifies the probability of developing negative effects related to functional and physical morbidity. [8]. A study developed at an ICU shows that early rehabilitation reduces hospital stay time and delirium, increases muscle strength and the patient's motivation. Long term effects were also observed, namely the increase of self-care ability, a faster return to independent functioning, improvement of physical function and reduced risk of readmission and death [9]. Another study, with ventilated patients who took part on an exercise program, showed that these patients improved their ability to perform DAs (hygiene, eating, transferring from bed to a chair and using wc) and increased walking distances in comparison with the group of control (not performing the exercises). There was an improvement of objective measures, such as the FIM and the Barthel Index, among the participant group at the time of hospital discharge [10]. For these reasons, assessing the level of dependence in selfcare is an important skill for a nursing professional, as it will allow to plan an individualized care and implement realistic interventions adjusted to the patient's needs, leading to feasible outcomes. Rehabilitation should, therefore, been seen as an enabler and promoter of the patient's function outcomes, as evidence suggests that structured rehabilitation processes can avoid patient's mobility deterioration [11-13].

Considering the current situation and based on data from the last census [14], the population presented in this article is characterized as an aged population presenting difficulties performing DAs $(20.85 \%)$ when compared with the national average $(17,8 \%)$. Walking is one of the main limitations [14]. This way and to respond to the needs of patients at a Stroke and Intensive Care Units of a Central Hospital in the interior of Portugal, we propose to organize an intervention plan (IP) focused on mobility issues. Taking into account Rehabilitation Nursing diagnoses we aim to design a specific and individualized intervention plan that aims autonomy capacity building. The intervention plan targeted people with mobility deficits and included bed mobility and transfers, which are considered pillars for developing long term patients' autonomy. 


\section{Methods}

The development of the IP is included in the Nursing Master's degree, Rehabilitation Field. Its implementation was previously authorized by the Central Hospital. The participants signed a voluntary and informed consent and ethical principles, data anonymity and confidentiality were respected.

The IP takes place within a research - action context and is based on participation and action as the researcher interacts with the individuals and can serve as a facilitator for behavioural change [15]. The cross-sectional plan took place between 14th October and 14th December in 2017. The selected patients were the ones admitted at the Stroke Unit and ICU and the inclusion criteria were the following: admission period at the unit inferior to $48 \mathrm{~h}$; to show commitment with mobility; to be collaborative; to accept to participate in the project by signing the required consent; possibility to be part of the intervention plan for at least 3 days. The target population was 50 patients (n), 41 were excluded as they did not fulfil the required criteria. The sample was composed by 9 patients (n), 6 patients admitted at the Stroke Unit and 3 patients at the ICU.

It was created a data collection protocol, composed by two distinct parts. The first, characterization variables (age, sex, medical diagnoses, family unit and carer presence), the assessment of the level of conscience (Glasgow Coma Scale) and of the patient's function capacity (Functional Independence Measure - FIM score). The second, presented information related with the focuses Bed Mobility and Transfers. For the latter, direct observation was used for assessing the necessary variables and obtaining a RN diagnosis. Each focus group included 3 variables, with defined and sustained criteria according to standard documentation about nursing care for rehabilitation nursing [16]. The change in one of the criteria (Table 1) suggests making the diagnosis and implement the planned interventions.

Table 1. Diagnoses evaluation grid

\begin{tabular}{|c|c|c|}
\hline Focus & Variable & Diagnoses criteria \\
\hline \multirow[t]{3}{*}{ Bed mobility } & Muscle Tone & $\begin{array}{l}\text { Shows contractures } \\
\text { Shows an increased tone } \\
\text { Shows joint stiffness } \\
\text { Presence of Spasticity } \\
\text { Presence of Hypotonia } \\
\text { Modified Ashworth Scale }\end{array}$ \\
\hline & Muscle Movement & $\begin{array}{l}\text { Has movement control } \\
\text { Has reduced movement } \\
\text { Mobilizes actively }\end{array}$ \\
\hline & Positioning & $\begin{array}{l}\text { Can position him/herself } \\
\text { Knows the adaptation technique for positioning } \\
\text { Braden Scale }\end{array}$ \\
\hline
\end{tabular}

(continued) 
Table 1. (continued)

\begin{tabular}{|c|c|c|}
\hline Focus & Variable & Diagnoses criteria \\
\hline \multirow[t]{3}{*}{ Transferring } & Body Balance & $\begin{array}{l}\text { Has seated static balance } \\
\text { Has seated dynamic balance } \\
\text { Has static orthostatic seated balance } \\
\text { Has dynamic orthostatic seated balance } \\
\text { Has vicious postures } \\
\text { Supports his/her own weight in different positions } \\
\text { Berg Scale }\end{array}$ \\
\hline & Transfers & $\begin{array}{l}\text { Transfers from lying to sitting position } \\
\text { Transfers from sitting to standing } \\
\text { Needs an auxiliary device for transferring } \\
\text { Knows the adaptation technique for transferring }\end{array}$ \\
\hline & Standing & $\begin{array}{l}\text { Can lift the SMs } \\
\text { Can lift the IMs } \\
\text { Supports his/her own weight while standing } \\
\text { Is alert for security cautions while lifting } \\
\text { Needs an auxiliary device for standing }\end{array}$ \\
\hline
\end{tabular}

For Bed Mobility the following variables were evaluated: muscle tone, muscle movement and positioning. The focus 'Transfers' included the variables body balance, standing and transfers. To support the evaluation, valid and objective measures were used, namely the Modified Ashworth Scale, the Berg Scale and also the Braden Scale.

Regarding the procedure, the evaluation was led in 2 different moments. In the first stage, the Initial Evaluation (T1) took place when the patient was first approached and in the second stage it took place by the end of the third day of intervention (T2). In general, the following stages were identified: Stage A - Evaluation - feedback was given for each variable (when changes occurred or not). When they were evaluated as positive, interventions were implemented. The Stage A coincides with the T1 moment. Stage B - Intervention - The Intervention Plan was composed by a set of exercises that aimed bed mobility and transfers. It included joint mobility, standard antispastic positioning, practising balance sitting in bed and in a chair. The intervention plan was adapted to each patient needs, with types of mobility and exercises appropriated to the patient's situation, with his/her cooperation. Stage C - Reevaluation - after three consecutive days of interventions of $\mathrm{RN}$, the nursing diagnosis was reevaluated and the results presented. The reevaluation stage occurs during the $\mathrm{T} 2$ moment.

The data were inserted in a database and descriptive statistics was used to analyse them.

\section{Results}

The sample used in the IP was composed by 9 participants between 43 and 86 years old, from which we highlight the 60-69 years age group $(n=4)$. The majority were males $(n=6)$. The most common group was participants living with the spouse $(56 \%)$ 
and none of the participants had an informal carer at the time of the initial evaluation $(n=9)$. The diagnoses most observed were stroke $(n=7), 78 \%$.

Regarding the level of conscience (Glasgow Coma Scale), the patients at the Stroke Unit had an average score of 15, whereas the patients at ICU presented an inferior average value, score $=11$. Concerning functionality, a relatively low FIM score average was observed $(54,78)$ : there was a change on the patients' level of dependence and they needed assistance for performing at least 50\% DA's tasks. Moreover, significant differences in the FIM score were also observed in patients of both services. Patients at the Stroke Unit presented an average score of 69 in opposition with an average score of 26 for patients at ICU, who had a change in their level of dependence and needed more assistance in $25 \%$ of the tasks compared with the patients at the Stroke Unit. In general, the total sample showed positive results regarding the FIM score between the moments T1 and T2, as presented in Fig. 1.

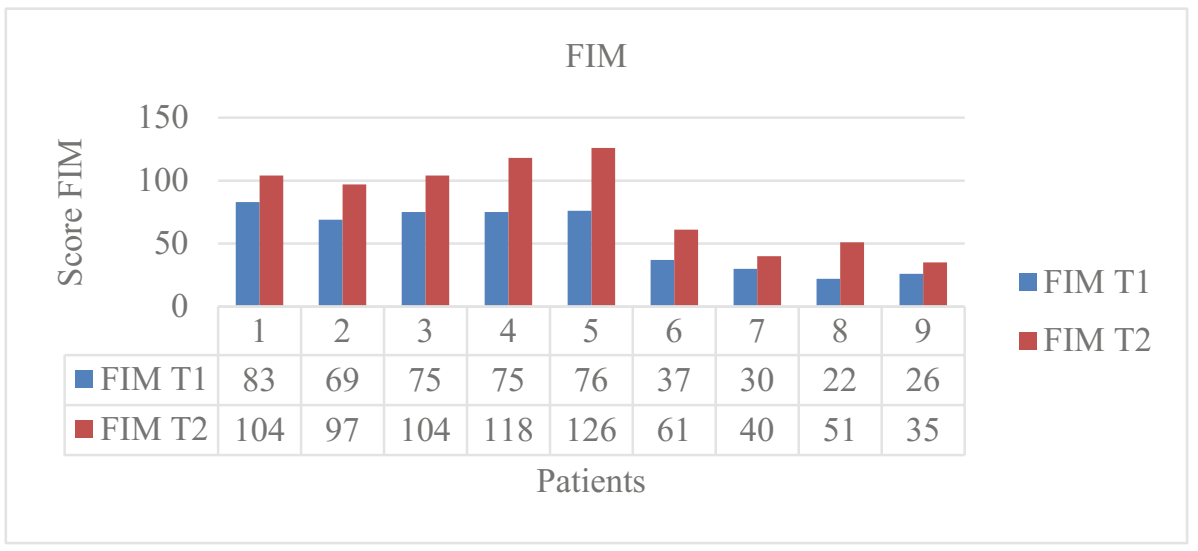

Fig. 1. Function Evolution - FIM

After the initial evaluation (T1), there were 6 associated nursing rehabilitation diagnoses, namely: Presence of hypertonia $(\mathrm{n}=1)$, Decreased muscle function $(\mathrm{n}=9)$, Potential to improve knowledge about the adaptation technique for positioning $(\mathrm{n}=9)$, Compromised body balance $(\mathrm{n}=9)$, Compromised standing $(\mathrm{n}=9)$ and Compromised adaptation technique for transferring $(\mathrm{n}=9)$ ). Looking at the results, there was only one patient diagnosed with hypertonia and the rest of the patients presented all the others diagnoses. Considering the interventions appropriated for each diagnoses and adapted to each patient, at T2 moment the diagnoses initially observed remained. However, there was a decrease in their prevalence. Therefore, in T2 the following could be observed: Presence of hypertonia $(\mathrm{n}=1)$, Decreased muscle function $(\mathrm{n}=4)$, Potential to improve knowledge about the adaptation technique for positioning $(\mathrm{n}=1)$, Compromised body balance $(\mathrm{n}=4)$, Compromised standing $(\mathrm{n}=3)$ and Compromised adaptation technique for transferring $(\mathrm{n}=4)$. Regarding objective measures, namely the risk of PU (Braden Scale) and balance (Berg Scale), the patients showed clear improvements in $\mathrm{T} 2$ in comparison with $\mathrm{T} 1$, as it is presented in Figs. 2 and 3. 




Fig. 2. Risk of PU (Braden Scale)

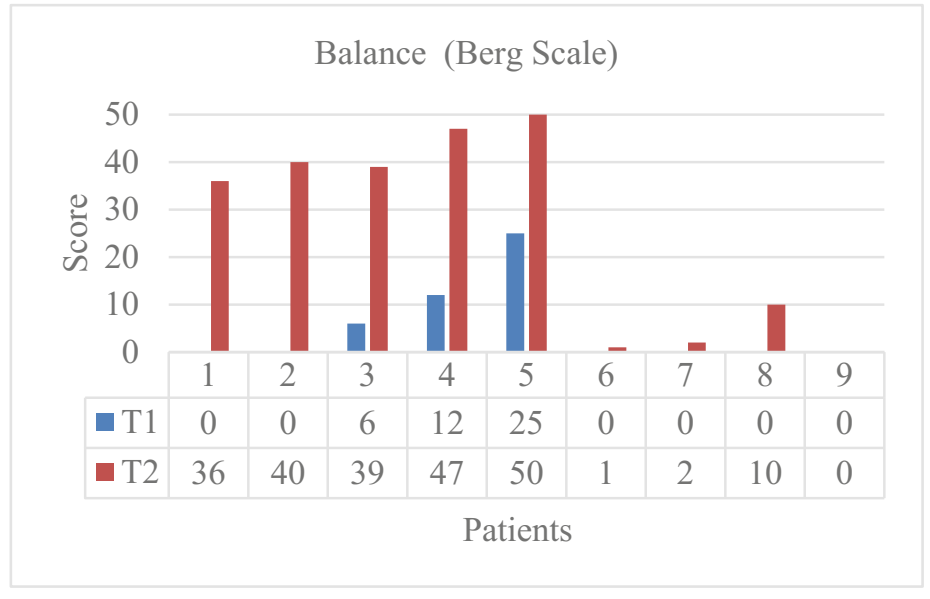

Fig. 3. Balance (Berg Scale)

After implementing the designed IP and after the participant patients' final evaluation (T2), there was approximately a reduction of 29 diagnosis of $\mathrm{RN}$ in comparison with T1, which means that $63 \%$ the diagnoses were solved. These data are illustrated in Table 2. 
Table 2. Rehabilitation nursing diagnoses

\begin{tabular}{|c|c|c|c|c|c|c|c|c|c|c|c|c|c|c|c|c|c|c|c|c|}
\hline Patient & 1 & & 2 & & 3 & & 4 & & 5 & & 6 & & 7 & & 8 & & 9 & & Tot: & \\
\hline Evaluation & $\mathrm{T}_{1}$ & $\mathrm{~T}_{2}$ & $\mathrm{~T}_{1}$ & $\mathrm{~T}_{2}$ & $\mathrm{~T}_{1}$ & $\mathrm{~T}_{2}$ & $\mathrm{~T}_{1}$ & $\mathrm{~T}_{2}$ & $\mathrm{~T}_{1}$ & $\mathrm{~T}_{2}$ & $\mathrm{~T}_{1}$ & $\mathrm{~T}_{2}$ & $\mathrm{~T}_{1}$ & $\mathrm{~T}_{2}$ & $\mathrm{~T}_{1}$ & $\mathrm{~T}_{2}$ & $\mathrm{~T}_{1}$ & $\mathrm{~T}_{2}$ & $\mathrm{~T}_{1}$ & $\mathbf{T}_{2}$ \\
\hline $\begin{array}{l}\text { Presence of } \\
\text { hypertonia }\end{array}$ & - & - & - & - & - & - & - & - & - & - & $x$ & $\mathrm{x}$ & - & - & - & - & - & - & 1 & 1 \\
\hline $\begin{array}{l}\text { Decreased muscle } \\
\text { function }\end{array}$ & $\mathrm{x}$ & $\mathrm{x}$ & $\mathrm{x}$ & - & $\mathrm{x}$ & $\mathrm{x}$ & $\mathrm{x}$ & - & $\mathrm{x}$ & - & $\mathrm{x}$ & $\mathrm{x}$ & $\mathrm{x}$ & $\mathrm{x}$ & $\mathrm{x}$ & - & $\mathrm{x}$ & - & 9 & 4 \\
\hline $\begin{array}{l}\text { Potential to } \\
\text { improve } \\
\text { knowledge about } \\
\text { the adaptation } \\
\text { technique for } \\
\text { positioning }\end{array}$ & $\mathrm{x}$ & - & $\mathrm{x}$ & - & $\mathrm{x}$ & - & $\mathrm{x}$ & - & $\mathrm{x}$ & - & $\mathrm{x}$ & $\mathrm{x}$ & $\mathrm{x}$ & - & $\mathrm{x}$ & - & $\mathrm{x}$ & $\mathrm{x}$ & 9 & 1 \\
\hline $\begin{array}{l}\text { Compromised } \\
\text { body balance }\end{array}$ & $\mathrm{x}$ & - & $\mathrm{x}$ & $\mathrm{x}$ & $\mathrm{x}$ & - & $\mathrm{x}$ & - & $\mathrm{x}$ & - & $\mathrm{x}$ & $\mathrm{x}$ & $\mathrm{x}$ & $\mathrm{x}$ & $\mathrm{x}$ & - & $\mathrm{x}$ & $\mathrm{x}$ & 9 & 4 \\
\hline $\begin{array}{l}\text { Compromised } \\
\text { standing }\end{array}$ & $\mathrm{x}$ & - & $\mathrm{x}$ & - & $\mathrm{x}$ & - & $\mathrm{x}$ & - & $\mathrm{x}$ & - & $\mathrm{x}$ & $\mathrm{x}$ & $\mathrm{x}$ & $\mathrm{x}$ & $\mathrm{x}$ & - & $\mathrm{x}$ & $\mathrm{x}$ & 9 & 3 \\
\hline $\begin{array}{l}\text { Compromised } \\
\text { adaptation } \\
\text { technique for } \\
\text { transfers }\end{array}$ & $\mathrm{x}$ & - & $\mathrm{x}$ & - & $\mathrm{x}$ & - & $\mathrm{x}$ & - & $\mathrm{x}$ & - & $\mathrm{x}$ & $\mathrm{x}$ & $\mathrm{x}$ & $\mathrm{x}$ & $\mathrm{x}$ & - & $\mathrm{x}$ & $\mathrm{x}$ & 9 & 4 \\
\hline RN Diagnoses (n) & 5 & 1 & 5 & 1 & 5 & 1 & 5 & $\mathbf{0}$ & 5 & $\mathbf{0}$ & 6 & 6 & 5 & 4 & 5 & $\mathbf{0}$ & 5 & 4 & 46 & 17 \\
\hline $\begin{array}{l}\text { Solved RN } \\
\text { Diagnoses (n) }\end{array}$ & 4 & & 4 & & 4 & & 5 & & 5 & & $\mathbf{0}$ & & 1 & & 5 & & 1 & & $\begin{array}{l}29 \\
(63\end{array}$ & \\
\hline
\end{tabular}

\section{Discussion}

While characterizing the sample, we observed that the participants' average age was 65 years old, with a predominance of males $67 \%(n=6)$. Similar data to the ones collected by the 'Rede Médicos Sentinela' referring to the year 2015 [17], where the highest stroke incidence rate was observed in males in the age group of 75 years old or more. The fact that the sample group didn't have informal carers reveals the level of independence of these patients performing DAs before the illness.

Regarding the functionality, a change in the patients' FIM score after the intervention plan (T2) was observed. Results that are congruent with the study developed by Kinoshita et al. [18], where the FIM score in patients with acute stroke before and after the intervention plan were evaluated. The results showed that functionality (FIM score) improved significantly in the three groups of the study and the gains (FIM) were more evident in the group that initiated the program within the first $24 \mathrm{~h}$. The authors concluded that an intervention plan was valuable and helped the patients with acute stroke to improve their ability for performing DAs.

As referred previously, after T2, the RN diagnoses were still observed but with decreased prevalence. We remind that most patients presented a stroke diagnosis. Depaul, Moreland and Dehueck [19], reinforce the idea that this type of population has great needs related to mobility. However, keeping in mind the duration of the intervention plan, a significant number of $\mathrm{RN}$ diagnoses were solved in $\mathrm{T} 2$. 
Referring specifically to the variable of muscle tone, presence of hypertonia, we realized that the short duration of the IP could have had influence on its evolution, revealing that it is insufficient for this variable. Studies in this area present durations of 6 weeks or more [20]. However, the aggravation of the patient's level of hypertonia was not observed. Such fact reveals the need to keep implementing the IP focusing more on standard antispastic body positioning.

Benefits for the other diagnosis were also observed: Decreased Muscle Function, Potential to improve knowledge about the adaptation technique for positioning, Compromised body balance, Compromised standing and Compromised adaptation technique for compromised for transferring. 63\% of the RN diagnoses were solved $(\mathrm{n}=29)$. Studies show that such interventions result in benefits for patients' mobility, as they favour the functional recovery in the acute phase and improve the quality of life of the patient after being discharged. The passive, active mobilization or the combination of both is associated with increased muscle strength, less days of in-hospital stay, less duration of delirium, less days of invasive mechanical ventilation dependence, higher probability to walk without medical assistance after discharge and a better functional status when discharged [21, 22]. Moreover, provide care to positioning also reveals to result positively regarding passive mobility and comfort [23]. Considering the developed IP, we observe that taking care of the positioning led to the reduction of risk of developing UP.

An intervention plan focused on body balance commitment can become valuable. After four weeks of study, Moghe and Kanase [24], concluded that initial interventions for controlling the trunk are effective and improved the balance in individuals with stroke and that, together with structured exercises, they contribute for a faster recovery in acute and subacute stages of stroke [24]. Buyukavci et al. [25], obtained similar results. Conventional exercises, or these combined with exercises of trunk balance, can result in significant balance, functional condition and ambulation improvements [25]. The IP sample group showed identical results after its implementation. By evaluating with the Berg Scale, we observe in T1 a total of 6 patients with a score $=0$ and in $\mathrm{T} 2 \mathrm{a}$ global improvement of the body balance, although with low scores.

As for the variable standing, it was observed that improving muscle function and balance was crucial for the gains obtained in the IP in T2 and, therefore, we argue for the importance of the IP regarding this variable. This is also present in Thomsen, Snow, Rodriguez and Hopkins [26], study. The aim of the study was to increase the patients' ambulation with acute respiratory failure, who were transferred from different ICU to a specific ICU, where the activity was focused on care. The mobility activities began in the first $24 \mathrm{~h}$ until the moment of discharge. The patients were submitted to an early action protocol, where interventions such as early standing and practising standing took place. In the first $24 \mathrm{~h}$ the authors observed an increase of the percentage of patients sitting in bed, sitting in a chair and of the ones who started walking [26]. Morris et al. [27], also corroborate these conclusions in their study. The authors divided two groups of patients in an ICU. One group was submitted to a mobility protocol and the other only to medical general care. The results demonstrated that patients under the mobility protocol started to stand earlier than the other group, within a difference of 5 days [27].

The difficulty with transfers motivated Alexander, Grunawalt, Carlos and Augustine [28], to design a set of exercises for bed mobility to test key movements, such as 
arms, legs and trunk movements that could probably contribute to the success of moving from one place to another. The study suggested that to improve bed mobility in older adults, the exercises should go beyond the improvement of the trunk function and therefore more exercises for bed mobility and training about the superior members positioning should been included, as these would be essential for straightening the trunk [28]. The IP results suggest that the integration of the exercises mentioned above was essential for the gains observed in the ability of the patient to go from the lying position to the seated position and from this to standing.

\section{Conclusion}

The effectiveness of the IP was evidenced by the Nursing Rehabilitation diagnoses being solved and reduced. Data suggest that the implementation of an IP focusing on people with mobility deficits decreased their level of dependence. We realised that when improving functional mobility, we are improving indirectly other variables, namely body balance, ability to transfer and the risk for developing UP. To improve muscle function and balance, implement exercises and provide information to the patient about procedures were crucial factors that enabled the patient to stand, easing the transfers and contributing to their independence.

Considering the results obtained, we believe that small evolutions should not be underestimate as, in the long run, they can result in great gains. It is not enough to evidence the partial gains, but also the gains that will contribute for self-care capacity building and for the highest possible level of independence.

We believe that the protocol for evaluating functionality, namely mobility, should be made available electronically. This allows the evaluations to be carried out by remote access, from the place where the evaluator is located. We then have the conditions to obtain nursing diagnoses, while keeping a history that traces the care process.

\section{References}

1. Booth, K., et al.: Progressive mobility protocol reduces venous thromboembolism rate in trauma intensive care patients: a quality improvement project. J. Trauma Nurs. Official J. Soc. Trauma Nurses 23(5), 284-289 (2016)

2. Kneafsey, R.: A systematic review of nursing contributions to mobility rehabilitation: examining the quality and content of the evidence. J. Clin. Nurs. 16, 325-340 (2007)

3. Hernández, B., Benjumea, P., Tuso, L.: Indicadores del desempeño clínico fisioterapéutico en el manejo hospitalario temprano del accidente cerebrovascular (ACV). Revista Ciencias De La Salud 11(1), 7-34 (2013)

4. Svendsen, M., Ehlers, L., Hundborg, H., Ingeman, A., Johnsen, S.: Processes of early stroke care and hospital costs. Int. J. Stroke Official J. Int. Stroke Soc. 9(6), 777-782 (2014)

5. Kutlubaev, M., Akhmadeeva, L.: The early post-stroke mobilization. Vopr. Kurortol. Fizioter. Lech. Fiz. Kult. 92(1), 46-50 (2015)

6. Oliveira, A., Araújo, T., Costa, A., Morais, H., Silva, V., Lopes, M.: Evaluation of patients with stroke monitored by home care programs. Revista da Escola de Enfermagem da USP 47(5), 1143-1149 (2013) 
7. Schmidt, U., Knecht, 1., MacIntyre, M.: Should early mobilization be routine in mechanically ventilated patients? Respir. Care 61(6), 867-875 (2016)

8. Azevedo, P., Gomes, B.: Efeitos da mobilização precoce na reabilitação funcional em doentes críticos: uma revisão sistemática. Revista de Enfermagem Referência IV(5), 129-138 (2015)

9. Hopkins, R., Mitchell, L., Thomsen, G., Schafer, M., Link, M., Brown, S.M.: Implementing a mobility program to minimize post-intensive care syndrome. AACN Adv. Crit. Care 27(2), 187-203 (2016)

10. Schweickert, W., et al.: Physical and occupational therapy in mechanically ventilated, critically ill patients: a randomised controlled trial. Lancet 373(9678), 1874-1882 (2009)

11. Mudge, A., Giebel, A., Cutler, A.: Exercising body and mind: an integrated approach to functional independence in hospitalized older people. J. Am. Geriatr. Soc. 56(4), 630-635 (2008)

12. Said, C., Morris, M., Woodward, M., Churilov, L., Bernhardt, J.: Enhancing physical activity in older adults receiving hospital based rehabilitation: a phase II feasibility study. BMC Geriatr. 12, 26 (2012)

13. Folden, S., Tappen, R.: Factors influencing function and recovery following hip repair surgery. Orthop. Nurs. 26(4), 234-241 (2007)

14. Instituto Nacional de Estatística: Censos 2011 Resultados Definitivos - Região Alentejo (2012). http://censos.ine.pt/ngt_server/attachfileu.jsp?look_parentBoui=156656957\&att_ display=n\&att_download=y. Accessed 01 Oct 2018

15. Carvalho e Silva, J., Ribeiro de Morais, E., Figueiredo, M., Tyrrell, M.: Pesquisa-acção: concepções e aplicabilidade nos estudos em Enfermagem. Rev. Bras. Enferm. 64(3), 592-595 (2011)

16. Ordem dos Enfermeiros: Padrão Documental dos Cuidados de Enfermagem da Especialidade de Enfermagem de Reabilitação. Colégio de Especialidade de Enfermagem de Reabilitação, Porto, pp. 1-60 (2015). http://www.ordemenfermeiros.pt/colegios/Documents/ 2015/MCEER_Assembleia/PadraoDocumental_EER.pdf. Accessed 01 Oct 2018

17. Rodrigues, A.P., Batista, I., Sousa-Uva, M., Pereira, S.: Médicos-sentinela: o que se fez em 2015. Instituto Nacional de saúde Doutor Ricardo Jorge, IP, Lisboa (2016)

18. Kinoshita, T., et al.: Effects of physiatrist and registered therapist operating acute rehabilitation (PROr) in patients with stroke. PLoS ONE 12(10), e0187099 (2017)

19. Depaul, V.P., Moreland, J.D., Dehueck, A.L.: Physiotherapy needs assessment of people with stroke following discharge from hospital, stratified by acute functional independence measure score. Physiotherapie Can 65(3), 204-214 (2013)

20. Krishnamoorthy, K., Varadharajulu, G., Kanase, S.: Effect of close kinematic chain exercises on upper limb spasticity in hemiparetic adult. Indian J. Physiother. Occup. Ther. 11(2), 146-152 (2017)

21. Tipping, C., et al.: The effects of active mobilisation and rehabilitation in ICU on mortality and function: a systematic review. Intensive Care Med. 43(2), 171-183 (2017)

22. Rosa, D., Olgiati, T.: Revisione narrativa della letteratura sulla mobilizzazione precoce in corso di ventilazione meccanica (A narrative review of early mobilization during mechanical ventilation). SCENARIO Off. Italian J. ANIARTI 34(3), 32-38 (2017)

23. Pickenbrock, H., Ludwig, V.U., Zapf, A., Dressler, D.: Conventional versus neutral positioning in central neurological disease: a multicenter randomized controlled trial. Deutsches Arzteblatt Int. 112(3), 35-42 (2015)

24. Moghe, D.M., Kanase, S.: Effect of early intervention for trunk control in stroke patients. Indian J. Physiother. Occup. Ther. 11(3), 177-182 (2017) 
25. Buyukavci, R., Şahin, F., Sağ, S., Doğu, B., Kuran, B.: The impact of additional trunk balance exercises on balance, functional condition and ambulation in early stroke patients: randomized controlled trial. Turkish J. Phys. Med. Rehabil. 62(3), 248-256 (2016)

26. Thomsen, G., Snow, G., Rodriguez, L., Hopkins, R.: Patients with respiratory failure increase ambulation after transfer to an intensive care unit where early activity is a priority. Crit. Care Med. 36(4), 1119-1124 (2008)

27. Morris, P., et al.: Early intensive care unit mobility therapy in the treatment of acute respiratory failure. Crit. Care Med. 36(8), 1-8 (2008)

28. Alexander, N.B., Grunawalt, J.C., Carlos, S., Augustine, J.: Bed mobility task performance in older adults. J. Rehabil. Res. Dev. 37(5), 633-638 (2000) 